\title{
Intranasal Premedication Combined with Erector Spinae Plane Block during Spinal Surgery for a Paediatric Patient: A Case Report
}

\section{Bao L, Qu M and Jing S*}

Department of Anesthesiology, The Second Affiliated Hospital, Third Military Medical University, Chongqing, People's Republic of China

*Correspondling author: Jing S, Department of Anesthesiology, The Second Affiliated Hospital, Army Medical University (Third Military Medical University) No. 83, Xinqiao Zheng Street, Shapingba District, Chongqing, China

Received: April 06, 2021; Accepted: J une 01, 2021; Published: J une 08, 2021

\begin{abstract}
Posterior spinal fusion surgery for congenital scoliosis may cause substantial surgical trauma. Erector Spinae Plane Block (ESPB) can alleviate pain in adults who undergo spinal surgery; however, reports regarding its use in paediatric patients are limited. Here, we report the case of a 2-year-old girl who underwent posterior spinal fusion for congenital scoliosis. The patient initially experienced severe sadness upon separation from her parents; however, after performing various interactive activities, premedication using intranasal dexmedetomidine and midazolam was administered to sedate the patient. Ultrasound-guided bilateral ESPB was applied for perioperative analgesia. The anaesthesiologist identified mild-to-moderate pain during the first 6 days after surgery using the visual analogue scale for pain and Face, Legs, Activity, Cry, and Consolability scale. Therefore, ESPB may provide a satisfactory analgesic effect and help reduce postoperative opioid consumption in children who require spinal fusion surgery.
\end{abstract}

Keywords: Erector spinae plane block; Anaesthesia; Paediatric patients; Spinal fusion; Congenital scoliosis

\section{Abbreviations}

CS: Congenital Scoliosis; ESPB: Erector Spinae Plane Block; FLACC: Face, Legs, Activity, Cry and Consolability; PSF: Posterior Spinal Fusion; VAS: Visual Analogue Scale

\section{Introduction}

Congenital Scoliosis (CS) involves scoliotic deformities originating from developmentally anomalous vertebrae [1]. Posterior Spinal Fusion (PSF) is a complex surgical treatment for CS that causes major surgical trauma. Traditional pain control strategies in this setting have adverse effects, which have directed significant attention to peripheral nerve block, given its better postoperative analgesic effects.

Erector Spinae Plane Block (ESPB), initially described as an interfacial plane block by Forero et al. in 2016 [2], effectively blocks the ventral rami of the spinal nerves and the dorsal rami that innervate the back [3]. ESPB effectively alleviates pain in adults undergoing spinal surgery; however, reports regarding its use in paediatric patients are limited. Furthermore, paediatric patients with CS may have concerns regarding surgical treatment, given the related trauma and pain, which makes perioperative anaesthesia and postoperative pain management crucial considerations. We report the case of a paediatric patient who underwent surgery for CS with bilateral bilevel ESPB which appeared to improve postoperative pain and reduce opioid consumption.

\section{Case Presentation}

Written informed consent for publication was obtained from the child's parents.
A 2-year-old girl (height, $0.84 \mathrm{~m}$; weight, $14 \mathrm{~kg}$ ) was diagnosed with CS. She began exhibiting signs of spinal deformity, including uneven shoulders and slight back pain (Figure 1); the deformity was at an early stage and was not affecting cardiac or pulmonary functions. Renal ultrasonography revealed congenital absence of the left kidney; however, electrocardiography, chest radiography, echocardiography, and laboratory test results were normal. Nevertheless, given the risks associated with CS, PSF was planned.

The patient initially experienced severe sadness upon separation from her parents. However, after performing various interactive activities, we were able to administer intranasal premedication using a 1-mL syringe (dexmedetomidine $(3 \mu \mathrm{g} / \mathrm{kg})$ and midazolam $(0.15 \mathrm{mg} /$ $\mathrm{kg})$ ) 30min before anaesthesia induction on the day of surgery. The child was sedated and subsequently transferred to the operation room. After routine monitoring, general anaesthesia was induced intravenously using propofol $(2 \mathrm{mg} / \mathrm{kg})$, sufentanil $(0.35 \mu \mathrm{g} / \mathrm{kg})$, and cisatracurium $(0.2 \mathrm{mg} / \mathrm{kg})$. Nasotracheal intubation was performed using an endotracheal tube (internal diameter, $5.0 \mathrm{~mm}$; insertion depth, $16 \mathrm{~cm}$ ). Ultrasound-guided punctures of the internal jugular and arteria radialis were performed, and the patient was supported with a fluid warming device and medical heating blanket.

The patient was then turned from the supine position to the lateral position, and ultrasound-guided (Mindray TE7) bilateral ESPB was applied at the T8 and L1 levels (Figure 2) by an experienced anaesthesiologist. Four injection points (total volume, $10 \mathrm{~mL}$ of $0.25 \%$ ropivacaine) were selected based on the extent of the planned incision and specific location of the spinal deformity. The needle was inserted through the erector spinae muscle to the specified transverse process using an in-plane approach, which produces an analgesic 


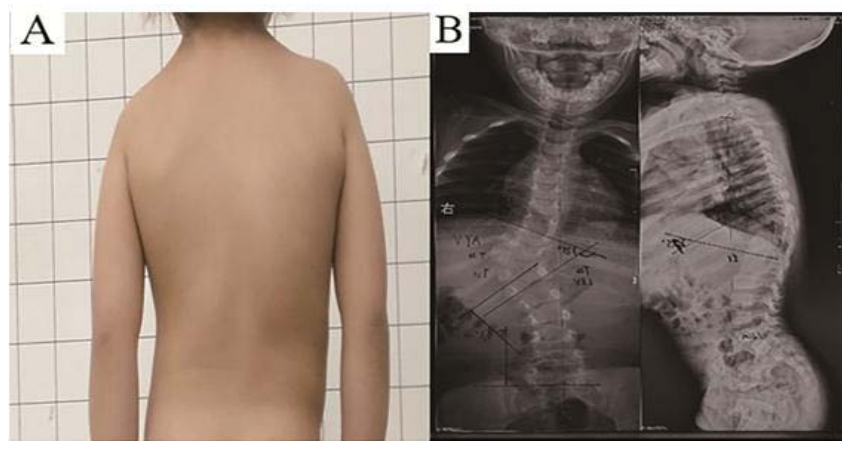

Figure 1: A) The patient's preoperative spinal deformity included uneven shoulders and scoliosis. B) Imaging findings.

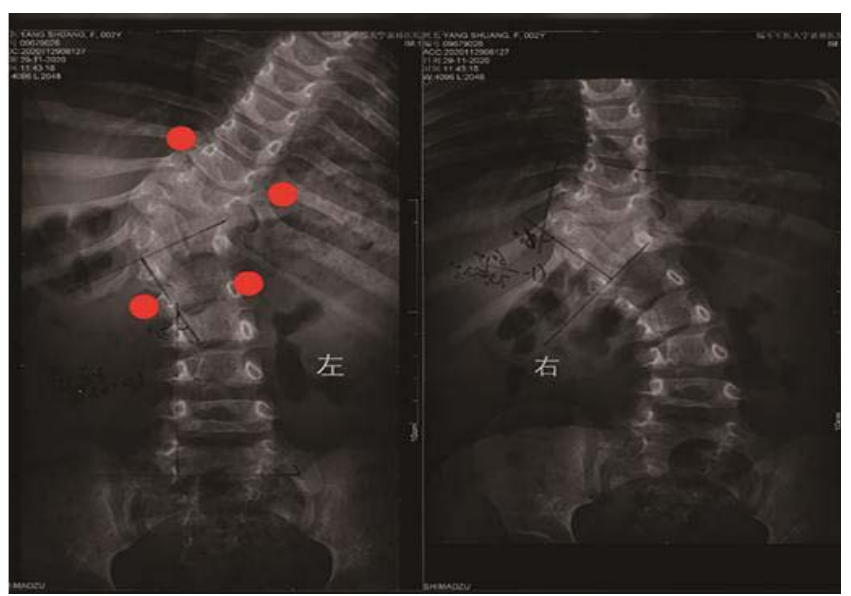

Figure 2: Ultrasound-guided erector spine plane block was performed at the T8 and L1 levels (red points).

affect because the local anaesthetic spreads within this potential space and acts on the spinal nerves at multiple levels. The patient exhibited $<10 \%$ increases in heart rate and blood pressure upon skin incision, which we believed confirmed the block's success. Given the surgical procedure's prolonged and extensive nature, we administered an additional intraoperative analgesic during the surgery (intravenous sufentanil at $0.2 \mu \mathrm{g} / \mathrm{kg}$ to a total dose of $3 \mu \mathrm{g}$ ). Controlled hypotension helped control bleeding during the nearly $6 \mathrm{~h}$ procedures; additionally, we monitored safety based on the somatosensory and motor-evoked potentials.

Postoperatively, the patient was transferred to the post-anaesthesia care unit, where we initiated patient-controlled intravenous analgesia ( $1 \mathrm{~mL}$ bolus of tramadol $(200 \mathrm{mg}$ ) and ramosetron $(0.6 \mathrm{mg})$ with a $15 \mathrm{~min}$ lock-out interval) and a $1 \mathrm{~mL}$ bolus was administered immediately. The patient recovered spontaneous and regular respiratory movements $\sim 40 \mathrm{~min}$ post-surgery and was awake and extubated calmly $\sim 30 \mathrm{~min}$ later. The patient reported no pain at the back incision upon tube extraction, although she verbalised pain to her parents $\sim 8 \mathrm{~h}$ postoperatively. The same anaesthesiologists monitored the patient for the first 6 postoperative days using the Visual Analogue Scale (VAS) for pain and Face, Legs, Activity, Cry, and Consolability (FLACC) scale, with 15 min of observation daily. During the first 4 postoperative days, the VAS revealed resting pain scores of $0-2 / 10$ and dynamic pain scores of 3-6/10. The FLACC scale scores were
$0-3 / 10$ at rest and 3-5/10 during routine care. However, the VAS and FLACC scale dynamic pain scores decreased substantially when the stimulations were removed. Given that the VAS and FLACC scores were both maintained at $0-3 / 10$, postoperative supervision was terminated after two additional days of monitoring on postoperative day 6 . The patient was subsequently switched to oral acetaminophen (0.1g per $12 \mathrm{~h})$, although she required patient-controlled intravenous analgesia a second time to alleviate pain. The total perioperative doses were $8 \mu \mathrm{g}$ for sufentanil and $400 \mathrm{mg}$ for tramadol.

\section{Discussion}

CS, the most common spinal scoliotic deformity in newborns, is identified in $\sim 1 / 1000$ live births, with a higher prevalence among girls (sex ratio, 2.5:1) [4]. These patients usually have other anomalies, and up to $20 \%$ of cases involve genitourinary anomalies (e.g. congenital absence of the left kidney, as in the present case). Patients often experience rapid CS progression during the first 5 years of life, which can affect heart and lung development. Herein, our patient's Cobb angle increased from $52^{\circ}$ to $73^{\circ}$ over a 3 -month period. Given the severe consequences of thoracic dysplasia and spinal cord compression, including the effects on cardiorespiratory function and the risk of paraplegia, most orthopaedic surgeons suggest early surgical treatment [4].

Perioperative anaesthesia management during PSF for CS is different for paediatric patients and adolescent/adult patients because surgery is a painful and often horrible experience for children, which makes it difficult to alleviate their anxiety and develop a friendly relationship. In the present case, the patient experienced severe sadness regarding separation from her parents. However, after various interactive activities and rewards for good behaviour, the girl permitted us to administer intranasal premedication with midazolam and dexmedetomidine. This intranasal premedication strategy is safe and effective and induces early and deep sedation because of midazolam's rapid effect and dexmedetomidine's prolonged effect, potentially making it easier to separate children from their parents [5]. Moreover, it is important to consider perioperative analgesia, as the median VAS scores are reportedly 5-7 on postoperative day 1 in cases of posterior spinal surgery for CS [6], highlighting the need for adequate perioperative analgesia [7]. Unfortunately, traditional pain management strategies, including opioids, nonsteroidal antiinflammatory drugs, and epidural local anaesthesia, have adverse effects, which has directed increasing attention to peripheral nerve block.

Although evaluating pain experienced by paediatric patients is critical, this is a difficult process. Lempinen et al. [8] described the feasibility and utility of the FLACC scale in evaluating pain among paediatric patients. Additionally, the VAS and FLACC scale scores are well correlated among children [9], a finding that generally agrees with our observations.

There is considerable interest in ESPB as an interfascial plane block, given its effective analgesic properties and widespread use. However, since the first report in 2016, there has been controversy related to its unclear mechanism of action. Studies suggest that the main underlying mechanisms for ESPB involve the local anaesthetic physically spreading over 3-6 vertebral levels in the ESP [10] and acting on the spinal nerves [11]. Furthermore, previous reports 
described ESPB as an effective and safe regional anaesthetic technique during abdominal, cardiothoracic, and hip surgeries; prostatectomies; and cervical spine fusions. A few cases involving ESPB during PSF surgery have been reported [12], although they involved adult not paediatric patients. A recent study of 70 children (2-18 years old) indicated that ESPB was safe, effective, and versatile in children [13], although that study did not specifically consider PSF surgery. To our knowledge, ours is the first report of ESPB during PSF surgery for CS in a paediatric patient. In the present case, after the ESPB, the patient experienced a good analgesic effect and required only small opioid doses with no clearly related complications. Given the postoperative infection risk in this patient population, we performed a singleinjection block that provides effective analgesia for at least 8-12 h [14]. Currently, a few experts have advised using dexmedetomidine plus ropivacaine to prolong the analgesia duration [15]; however, we recommended using ropivacaine alone because of concerns regarding neurotoxicity associated with the use of combined ropivacaine and dexmedetomidine [16].

The volume and concentration of the local anaesthetic play crucial roles in ESPB's clinical effects [11]. In adult patients, a single $20 \mathrm{~mL}$ injection of the local anaesthetic physically spreads over 3-6 vertebral levels in the ESP. We selected four injection points based on the extent of the planned incision and the expected physical spreading of the local anaesthetic, although there was limited information regarding the optimal volume and local anaesthetic concentration for ESPB in children. A small volume $(0.3-0.45 \mathrm{~mL} / \mathrm{kg}$ ) and low concentration (0.2-0.25\% ropivacaine) have been recommended for young children [11].

\section{Conclusion}

This case suggests that ESPB during PSF for CS may provide a satisfactory analgesic effect and help reduce postoperative opioid use in paediatric patients. Furthermore, intranasal premedication was an effective strategy for preparing the patient to be separated from her parents. The rational use of multiple methods, including different nerve block techniques and drug combinations, can help reduce related complications and accelerate the healing process in this setting.

\section{Acknowledgements}

The authors thank the staff at the Department of Anesthesiology, The Second Affiliated Hospital, Army Medical University (Third Military Medical University), for their cooperation.

\section{References}

1. Karaarslan UC, Gurel IE, Yucekul A, Demirkiran HG, Samdani A, Yilgor C, et al. Team approach: Contemporary treatment of congenital scoliosis. JBJS Rev. 2019; 7: e5.
2. Forero M, Adhikary SD, Lopez H, Tsui C, Chin KJ. The erector spinae plane block: A novel analgesic technique in thoracic neuropathic pain. Reg Anesth Pain Med. 2016; 41: 621-627.

3. Melvin JP, Schrot RJ, Chu GM, Chin KJ. Low thoracic erector spinae plane block for perioperative analgesia in lumbosacral spine surgery: A case series. Can J Anaesth. 2018; 65: 1057-1065.

4. Hedequist D, Emans J. Congenital scoliosis: A review and update. J Pediatr Orthop. 2007; 27: 106-116.

5. Chatrath V, Kumar R, Sachdeva U, Thakur M. Intranasal fentanyl, midazolam and dexmedetomidine as premedication in pediatric patients. Anesth Essays Res. 2018; 12: 748-753.

6. Gerbershagen HJ, Aduckathil S, van Wijck AJ, Peelen LM, Kalkman CJ, Meissner W. Pain intensity on the first day after surgery: A prospective cohort study comparing 179 surgical procedures. Anesthesiology. 2013; 118: 934-944.

7. Kwan MK, Chiu CK, Chan TS, Chong KI, Mohamad SM, Hasan MS, et al Trajectory of postoperative wound pain within the first 2 weeks following posterior spinal fusion surgery in adolescent idiopathic scoliosis patients. Spine (Phila Pa 1976). 2017; 42: 838-843.

8. Lempinen H, Pölkki T, Kyngäs H, Kaakinen P. Feasibility and clinical utility of the Finnish version of the FLACC pain scale in PICU. J Pediatr Nurs. 2020; 55: 211-216.

9. Crellin DJ, Harrison D, Santamaria N, Huque H, Babl FE. The psychometric properties of the FLACC scale used to assess procedural pain. J Pain. 2018; 19: 862-872.

10. Schoenfeldt J, Guffey R, Fingerman M. Cadaveric study investigating the mechanism of action of erector spinae blockade. Reg Anesth Pain Med. 2018; 43: 567-571.

11. Chin KJ, El-Boghdadly K. Mechanisms of action of the Erector Spinae Plane (ESP) block: A narrative review. Can J Anaesth. 2021; 68: 387-408.

12. Chin KJ, Lewis S. Opioid-free analgesia for posterior spinal fusion surgery using erector spinae plane (ESP) blocks in a multimodal anesthetic regimen. Spine (Phila Pa 1976). 2019; 44: E379-E383.

13. Holland EL, Bosenberg AT. Early experience with erector spinae plane blocks in children. Paediatr Anaesth. 2020; 30: 96-107.

14. Krishna SN, Chauhan S, Bhoi D, Kaushal B, Hasija S, Sangdup T, et al. Bilateral erector spinae plane block for acute post-surgical pain in adult cardiac surgical patients: A randomized controlled trial. J Cardiothorac Vasc Anesth. 2019; 33: 368-375.

15. Yao F, Xu S, Zhang W, Xiong H, Han J, Zhu A. Impacts of different administration modes of dexmedetomidine with $0.5 \%$ ropivacaine on intercostal nerve block. Ann Palliat Med. 2020; 9: 447-450.

16. Desai N, Kirkham KR, Albrecht E. Local anaesthetic adjuncts for peripheral regional anaesthesia: A narrative review. Anaesthesia. 2021; 76: 100-109. 